Article

\title{
A Systematic Review of the Use of Blockchain in Healthcare
}

\author{
Marko Hölb1 *(D), Marko Kompara (D, Aida Kamišalić (D) and Lili Nemec Zlatolas (D) \\ Faculty of Electrical Engineering and Computer Science, University of Maribor, Maribor 2000, Slovenia; \\ marko.kompara@um.si (M.K.); aida.kamisalic@um.si (A.K.); lili.nemeczlatolas@um.si (L.N.Z.) \\ * Correspondence: marko.holbl@um.si; Tel.: +386-2-220-7361
}

Received: 7 September 2018 ; Accepted: 6 October 2018 ; Published: 10 October 2018

\begin{abstract}
Blockchain technology enables a decentralized and distributed environment with no need for a central authority. Transactions are simultaneously secure and trustworthy due to the use of cryptographic principles. In recent years, blockchain technology has become very trendy and penetrated different domains, mostly due to the popularity of cryptocurrencies. One field where blockchain technology has tremendous potential is healthcare, due to the need for a more patient-centric approach to healthcare systems and to connect disparate systems and increase the accuracy of electronic healthcare records (EHRs). In this systematic review, an analysis of state-of-the-art blockchain research in the field of healthcare is conducted. The aim is to reveal the potential applications of the technology and to highlight the challenges and possible directions of blockchain research in healthcare. First, background information is discussed, followed by a description of the exact methodology used in this paper. Next, an analysis of the results is given, which includes a bibliometric overview, an analysis of gathered data and its properties, and the results of a literature quality assessment. Lastly, there is a discussion of the results from the analysis. The findings indicate that blockchain technology research in healthcare is increasing and it is mostly used for data sharing, managing health records and access control. Other scenarios are very rare. Most research is aimed at presenting novel structural designs in the form of frameworks, architectures or models. Findings also show that technical details about the used blockchain elements are not given in most of the analyzed publications and that most research does not present any prototype implementation or implementation details. Often even with a prototype implementation, no details about blockchain elements are given.
\end{abstract}

Keywords: blockchain; consensus; distributed systems; healthcare; systematic review

\section{Introduction}

Blockchain technology has been penetrating every aspect of Information and Communications Technology (ICT) and its use has been growing rapidly in recent years. The interest and development of this technology has primarily been driven by the enormous value growth of cryptocurrencies and large investments of venture capital in blockchain start-ups. It is forecast that the market for blockchain technology will grow until 2021 [1]. Currently there are more than 1500 crypto coins [2], which were created in just a few years after the introduction of Bitcoin [3]. Bitcoin was the pioneer digital coin. It assures that transactions are performed in a decentralized way without the need for a trusted central authority. As public keys are employed there is no need to disclose someone's identity. A vital part of the Bitcoin network are miners that receive coins for their computational work to verify and store transactions (payments) in the Bitcoin blockchain. The interested reader can find more information about Bitcoin in [4-6].

Cryptocurrencies are just one example of the use of blockchain technology. Basically there are three concepts that need to be distinguished in a cryptocurrency [4] — the blockchain, the protocol and 
the currency. A coin can implement its own currency and protocol, but use the blockchain of another coin like Bitcoin or Ethereum [7]. In the world of cryptocurrencies, the blockchain acts as a distributed ledger that stores all the performed transactions of coins. Con-sequentially, such a blockchain grows continuously as new blocks are added over time. Most known cryptocurrency blockchains are public and one can query their transactions through web platforms like blockchain.com-a web platform for querying the transactions of the Bitcoin blockchain.

Blockchain enables transactions between entities without the need for a (trusted) third-party. It relies on validators (often miners) that replace the third parties and validate transactions in a decentralized way. This is achieved through a distributed consensus-an ability to agree on something among multiple parties that do not trust each other. In the crypto-currency world, this computational problem is related to the double-spending problem, i.e., how to confirm that a specific amount of a digital coin has not already been spent without the validation of a trusted third-party (usually a bank), which keeps a record of all transactions and users' balances.

Surveys on the application of blockchain in different fields have already been published [8]. Zheng et al. [9-11] provide a review on the architecture and the different mechanisms involved in blockchain technology. Additionally, Karafiloski and Mishev [12], and Ahram et al. [13] provided overviews on blockchain technology with an emphasis on its application in big data and industrial applications. Conoscenti et al. [14] and Yli-Huumo et al. [15] published systematic reviews that analyze the application areas of blockchain and related research topics. There are papers that address the applicability of blockchain technology in the healthcare domain [16-25], but none of those took a systematic approach to review the target field.

\section{Motivation and Contribution}

In contrast to the mentioned papers, this work presents a systematic review and analysis of the state-of-the-art blockchain research in the field of healthcare. The aim of this paper is also to indicate the potential usage of blockchain in healthcare and to show the challenges and potential directions of blockchain research. This systematic review only includes research that introduces a new solution, algorithm, method, methodology, or architecture for the field of healthcare. Review type research, discussions of possible uses and applications of blockchain, and other non-relevant publications are excluded.

The remainder of this paper is organized as follows. In Section 2 we present background information on blockchain technology and its concepts as well as the need for blockchain in healthcare. The systematic review methodology, research questions, the included databases, selection of studies and the literature quality assessment are discussed in Section 3. Section 4 presents the data analysis including a bibliometric overview, an analysis of gathered data and its properties, and the literature quality assessment results. Furthermore, a discussion of the results is presented in Section 5 together with the discussion and answers to the research questions. The paper is summed up in Section 6 where conclusions are drawn and future work is discussed.

\section{Background}

In this section, we discuss the fundamentals of blockchain technology to help understand the rest of this paper.

A blockchain can be seen as a distributed ledger that shares data among a network of peers $[4,9,26]$. It was introduced together with Bitcoin and solved a long-lasting problem-the double-spend problem. In Bitcoin this is achieved through a consensus of the majority of the so-called mining nodes and the appending of valid transactions to the blockchain. The first application of blockchain was in cryptocurrency. However, it is not necessary to introduce a cryptocurrency in order to use blockchain and build decentralized applications [27].

To get the reader to better understand the concept of the blockchain, its basic characteristics and building elements will be described in the next subsections. 


\subsection{Blockchain—Distributed Ledger Technology}

A blockchain can be defined as a chain of blocks that are time-stamped and linked using cryptographic hashes. These blocks are sealed in a secure and immutable manner $[26,28]$. The chain is constantly growing and new blocks are being appended to the end, whereby each new block holds a reference (i.e., a hash value) to the content of the previous block [29]. The shareholders, also referred to as nodes of the blockchain are organized in a peer-to-peer (P2P) network. Each node in the network is in the possession of two keys [30]: a public key that is used for encrypting the messages sent to a node and a private key that is used to decrypt the messages and allows a node to read it. Thus the public key encryption mechanism is used to ensure the consistency, irreversibility and non-repudiability of a blockchain [9]. Only the proper private key can decrypt the messages encrypted with the corresponding public key. This concept is know as asymmetric cryptography. As a detailed explanation is out of the scope of this paper, further details can be found in [30,31].

All the blocks in the blockchain are linked using the so-called hash which is generated using a cryptographic one-way hash function (e.g., SHA256). It also ensures anonymity, immutability and compactness of the block [32].

Each transaction carried out by a node is signed before it is broadcasted to the network for later confirmation. The digital signing of a transaction using the private key enables authentication and provides integrity of a transaction. The first is due to the fact that only a user with a specific private key can sign the transaction and the second is due to the fact that an error during transmission of the data results in the inability of decryption (i.e., verifying a digital signature).

Transactions disseminated in the network and considered valid by the network are ordered and packed into time-stamped blocks by specific nodes, which are called miners when the network is using specific consensus mechanisms, such as proof-of-work or proof-of-stake. How the miners are chosen and what data is included in the block depend on the consensus protocol (a more detailed definition of a consensus protocol is given later). The blocks are then broadcast to the network, where the validation nodes verify that the received block contains valid transactions and that it references the previous block in the chain by using the corresponding hash. If both requirements are fulfilled, the nodes add the block to the blockchain. If the requirements are not fulfilled, the block is discarded.

This brings us to the role of network nodes. Since the blockchain network is a P2P network, a node can be regarded as a peer when it starts to connect and communicate with other nodes in the network, thus the appropriate name would be a peer-node. We will henceforth denote it as a "node" for easier comprehension. In layman's terms, a full node is any computer that has the core blockchain client installed and operates a full copy of the whole blockchain ledger [9,33,34].

A user wishing to engage with the blockchain connects to the blockchain network through a node [33]. The previously mentioned miners are a subset of nodes, since all miners must also operate a fully functional node. Hence, each miner is a node, but not every node is also a miner. This scenario is known from a specific public blockchain type employing the PoW (proof-of-work) consensus (more on this later in this section). Other types of blockchain networks, using other distributed consensus types, do not require mining, e.g., PoS (proof-of-stake) [15].

The basic tasks of a blockchain node are $[33,34]$ :

- connecting to the blockchain network,

- storing an up-to-date ledger,

- listening to transactions,

- passing on valid transactions into the network,

- listening for newly sealed blocks,

- validating newly sealed blocks - confirming transactions,

- creating and passing on new blocks. 


\subsection{Types of Blockchains}

Generally, there are different types of blockchains depending on the managed data, on the availability of such data, and on what actions can be performed by the user. These include:

- public permissionless,

- consortium (public permissioned),

- private.

All the data in the public permissionless (often called just public) blockchain is accessible and visible to the public. However, some parts of the blockchain could be encrypted in order to preserve a participant's anonymity [9]. In a public permissionless blockchain anyone is able to join the blockchain without any approval and can act as a simple node or as a miner (node). These types of blockchains are usually given an economic incentive, such as in cryptocurrency networks. Examples of such a blockchain include Bitcoin, Ethereum or Litecoin [3,7,35].

The consortium type blockchain enables only a selected group of nodes to participate in the distributed consensus process [9]. It can be used within one or across several industries. When a consortium blockchain is established within one industry (e.g., financial sector), it is opened for limited public use and partially centralized. On the other hand, a consortium between industries (e.g., insurance companies, financial institutions, governmental institutions) is opened for public use while still having established a partially centralized trust.

A private blockchain only enables chosen nodes to join the network. It is therefore a distributed yet centralized network [9]. Private blockchains are permissioned networks in order to control which nodes can perform transactions, execute smart contracts or act as miners. They are managed by one organization which is the trusted party. It is used for private purposes. Hyperledger Fabric [36] and Ripple [37] are examples of blockchain platforms that only support private blockchain networks. Note that such classifications are still being debated and different definitions might be found in literature.

A distinction between blockchains can also be made based on their purpose:

- for tracking digital assets (e.g., Bitcoin) and

- for running certain logic (i.e., smart contracts).

Some blockchains use tokens (e.g., Ripple, Bitcoin, Ethereum), while others do not (e.g., Hyperledger Fabric).

\subsection{Distributed Consensus Protocols}

For the blockchain network to remain functional, its peers need to agree on a certain state of the distributed ledger and on a way for packing data into blocks. Such an agreement is called a distributed consensus protocol and it validates the chronological order of generated transactions [9]. It ensures that a quorum of blockchain network peers agree on the precise state of the shared ledger, and thus the order in which new blocks are added to the ledger [26]. Some of the used distributed consensus protocols are $[9,26,38]$ :

- proof-of-work (PoW),

- proof-of-stake (PoS),

- delegated-proof-of-stake (DPoS),

- proof-of-importance (PoI),

- proof-of-activity (PoA),

- proof-of-burn (PoB),

- proof-of-deposit (PoD).

A distributed consensus protocol defines how a network determines which peer will prepare and seal the newest block with still unconfirmed and non-formatted data. The simplest way is to determine it randomly, but such an approach is not effective in terms of network longevity and can even be 
dangerous for the network, since peers could decide to attack the whole network [9]. The idea behind PoW, PoS and others is the fact that the chosen node (miner) contributes something valuable and the best node is rewarded. The reward promotes competition and a competition where adversaries check each others' work and valuables also mitigates the chances of a possible attack.

The PoW consensus protocol is used in the Bitcoin network. It uses computing power as a mechanism to determine the chosen peer $[3,9,15]$. The competition between peers is based on hashing unconfirmed transactions. Therefore, a peer's chance of being chosen is in proportion to its computational power. Each time a peer wins and is chosen, it gets a reward. The current reward in Bitcoin network is 12.5 newly generated bitcoins, which are added to the chosen peer's account [26]. The hashing competition, which is called mining, is based on the calculation of a block, containing unconfirmed transactions, a random nonce and a hash reference to the previous block. It is required that the outcome of the hash is equal to a predefined value. In case a miner reaches the required value, it broadcasts the newly generated block to the network. Other peers then validate it and if correct it is replicated in the network [9].

The PoS consensus protocol is based on the assets a peer possesses (i.e., the stake of the network value a peer has under control). A peer's chance of being chosen to confirm a new block is in proportion to its assets (i.e., wealth or stake). In practice, this is realized by having a peer deposit a predefined minimum number of its assets. This buys the node a ticket. The winner is chosen in a deterministic pseudo-random way from a group of peers with tickets. The competition in this case is not based on the computational power of the peers, meaning there is minimal energy consumption in comparison to the PoW. However, such an approach is similar to a shareholder corporation, where the rich have an advantage [38]. This works, because it is unlikely a peer will attack the network, since in this case it would attack its own assets [9]. There are multiple versions of the PoS consensus protocol, whereby each introduces a different approach on how to choose the validator in order to ensure fairness. One of these versions is the Distributed PoS (DPoS). The difference between a regular PoS and a DPoS system can be compared to the difference between a direct democracy and a representative democracy, since stakeholders vote in order to decide the signer, i.e., the delegate [9].

Therefore, we can state that nodes determine the distributed consensus, thus agreeing on specific rules and that the blockchain acts as a consensus mechanism to ensure that the nodes stay in sync [34].

\subsection{The Need for Blockchain in Healthcare}

One of the fields where blockchain is considered to have great potential is healthcare [39]. To transform healthcare, the focus should be given to the management of data that could benefit from the potential to connect disparate systems and increase the accuracy of EHRs. Blockchain technology can be used to support drug prescriptions and supply chain management, pregnancy and any risk data management as well as to support access control, data sharing and managing of an audit trail of medical activities. Other healthcare areas that can benefit from blockchain technology are provider credentials, medical billing, contracting, medical record exchange, clinical trials, and anti-counterfeiting drugs. Healthcare services are transforming to enable a patient-centric approach. Blockchain-based healthcare systems could enhance security and reliability of patients' data since patients would have control over their healthcare records. Those systems could also help consolidate patient data, enabling the exchange of medical records across different healthcare institutions.

Storing the medical data of patients is very important in healthcare. These data are very sensitive and therefore also a prime target for cyber-attacks. It is important to secure all sensitive data. Another aspect is control over data which would ideally be managed by the patient. Therefore, sharing and accessing the control of patients' healthcare data is another use case which can benefit from advanced modern technologies. Blockchain technology is very robust against attacks and failures, and provides different methods of access control. Therefore, blockchain provides a good framework for healthcare data. 
For personal medical data, the most adequate type of a blockchain would be a private blockchain. According to the Würst and Gervais decision model, a blockchain can be used in a scenario where multiple parties who do not trust each other need to interact and exchange common data, but would not like to involve a trusted third-party (TTP) [40]. Their model presents several factors which need to be considered when analyzing whether a specific scenario requires blockchain. Regarding storage, there are several factors (questions) that need to be considered [40]:

- Is there a need for storing data (with regard to the authors of a specific state)?

- Do you need multiple write access?

- Is a TTP available and can one that is always online be used?

Firstly, we have to determine the need for data storing (in a typical scenario this is a database). Subsequently, it needs to be determined if there is a need for writing access for multiple parties. If only one writer exists, then no blockchain is needed and alternative solutions can be considered (e.g., a database). It has to be noted that traditional databases offer better performance than a blockchain. If a TTP is available, is always online, and can be fully trusted, then there is no need for blockchain.

The Würst and Gervais decision model also helps determine what type of blockchain should be used (e.g., public permissionless, public permissioned, or private). If the writers are not known, the only obvious choice would be a public permissionless blockchain. If the TTP is offline, it could function similarly to a certification authority and the involved parties do not mutually trust each other, a permissioned blockchain could be employed. However, if all the parties mutually trust each other, then a database with shared access could be used instead of a blockchain. On the other hand, if writers are known and can be trusted, the choice falls between a public permissioned and private blockchain. The first is in the case where you need public verifiability and the second in the case when it is not needed. Please note that these parts of the model imply that the first three questions stated before are answered positively—otherwise deploying a blockchain is unnecessary.

The current medical data infrastructure mostly depends on trusted third parties. In several cases these cannot be fully trusted. The blockchain, which relies on consensus and does not need a central authority, is a possible solution to this problem.

\section{Methodology}

We first define the used methodology. We have limited our systematic review to the field of healthcare.

\subsection{Research Questions}

The study intended to answer the following research questions:

1. RQ1: To what extent is blockchain established in healthcare and how did this change over time?

2. RQ2: What are the current research trends for blockchain use in healthcare?

3. RQ3: What are the elements of blockchain technology used in the healthcare publications?

\subsection{Data Sources}

The systematic review included the following 9 electronic databases:

- Google Scholar,

- Clarivate Analytics-Web of Science (WoS),

- IEEE Xplore,

- PubMed,

- Medline EBSCO,

- Springer SpringerLink,

- Elsevier ScienceDirect,

- ACM Digital Library,

- Cochrane Central. 
The review was conducted by three reviewers and the search in all databases returned 6375 results. Due to a lack of advanced search options in Google Scholar and SpringerLink, they returned many non-related results. Therefore, we only included the first 200 most relevant results from these two databases.

The search for relevant publications was performed using the query string(s) defined below. The search strings were constructed based on the research domain and the defined research questions.

We have used the following search terms:

(blockchain OR "block chain") AND

(healthcare OR health OR medic* OR

medical OR medicine OR *health*)

In some cases where the ${ }^{*}$ symbol could not be used, we replaced the original search query with the following:

(blockchain OR "block chain') AND

(healthcare OR health OR medical OR

medicine OR m-health OR mhealth OR

ehealth OR e-health OR telehealth)

The search in the online digital libraries was conducted in June 2018. The search query was intentionally made as broad as possible in order to consider as many results related to the research questions posed in this systematic review as possible. The used procedure for searching and the selection of publications is summarized in Figure 1. The summary of the results returned for each database search are presented in Table 1.

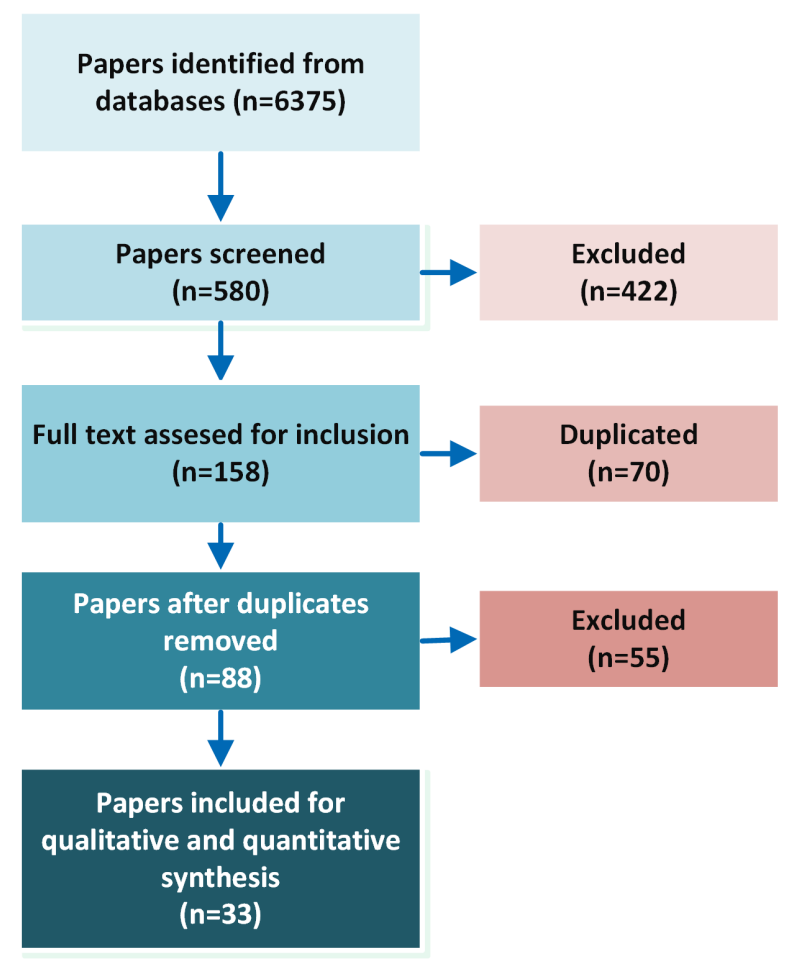

Figure 1. Flow diagram of the search. 
Table 1. Summary of Search Results.

\begin{tabular}{ccc}
\hline Database & Number of Results & Number of Results Suitable for Detailed Screening \\
\hline Google Scholar & $5500(200)$ & 53 \\
Web of Science (WoS) & 58 & 36 \\
IEEE Xplore & 49 & 22 \\
PubMed & 25 & 20 \\
Medline EBSCO & 30 & 16 \\
SpringerLink & $695(200)$ & 5 \\
Elsevier ScienceDirect & 9 & 3 \\
MDPI & 3 & 2 \\
ACM Digital Library & 6 & 1 \\
Cochrane Central & 0 & 0 \\
\hline Total & $\mathbf{6 3 7 5 ( 5 8 0 )}$ & $\mathbf{1 5 8}$ \\
\hline
\end{tabular}

\subsection{Selection of Studies}

The selection process started with 580 publications gathered from online digital libraries. Based on the criteria, the publications were either included in the systematic review or not. The selection process was divided into four phases:

- Phase 1: The search results were filtered according to the inclusion and exclusion criteria. The criteria are described in the systematic review protocol in Table A1 in Appendix A. We included studies from the years 2008 to 2019 (the last one due to the fact that some scientific publication already had issues set to be published in 2019). The reason for choosing the year 2008 as the beginning of the range is the introduction of Bitcoin in 2008 which was the first published application of the blockchain technology. Therefore, there are no blockchain related studies before 2008.

- Phase 2: We assessed and selected the results based on the relevance of the research questions by investigating only the title and abstract. We excluded 422 results.

- $\quad$ Phase 3: The duplicates from 9 different databases were removed. There were 158 publications found and after removing the duplicates, 88 publications were left for the next phase.

- Phase 4: The remaining results were inspected in more detail by a full reading. The analyzed publication had to relate closely to the defined research questions. The remaining results also had to include a novel and sufficient contribution to the field of healthcare by the means of a blockchain. Many results were excluded as the introduced idea was very general and no further details on its design or implementation were given. A further 55 results were excluded.

The final number of included publications in the systematic review was 33 .

The process itself was strict in order to ensure that only relevant and high-quality studies were considered.

The process of gathering, selecting and reviewing used in this review was controlled using the CASP Systematic Review Checklist [41], which addresses the assessment of research in systematic reviews.

\subsection{Data Gathering}

After collecting the relevant publications, we gathered data from those 33 publications. We started with basic information about the publications: title, author(s), publication type (e.g., conference proceeding, journal paper, etc.), publication year, and the number of citations based on Google Scholar Citations [42].

After collecting the basic information, we were interested in the two primary fields of interest in this paper-blockchain and healthcare.

We collected the contributions of the analyzed publications. Typical contributions are a presentation or discussion of a structural design (i.e., a framework or an architecture), an algorithm or a protocol, a consensus algorithm or a new metric. 
We were also interested for which purpose or field a blockchain included in healthcare was used. As blockchain technology can serve multiple purposes we have allowed for more than one to be selected. We found that a blockchain is usually used in the following fields:

- data sharing,

- access control,

- health records (EHRs, EMRs and PHRs),

- managing an audit trail,

- supply chain,

- $\quad$ others.

Many publications discuss record keeping. The majority in the form of EHRs, while the minority propose or discuss electronic medical records (EMRs) and personal health records (PHRs). In this paper we combine these into the field of health records.

We have also investigated if publications present an existing solution in use or just propose a concept with the potential for real-life application (with a possible proof of concept implementation).

Next, we identified details about blockchain elements that can be extracted from the analyzed publications. From the contents of the publications, we identified a blockchain platform (e.g., Ethereum, Hyperledger Fabric, etc.), a consensus algorithm (e.g., PoW, PoS, etc.), and a blockchain type (as defined in Section 2.2). Finally, we identified if the proposed solutions incorporated smart contracts. Any information not found in the publications was marked as "not defined".

\subsection{Literature Quality}

We have also conducted an assessment of literature quality. To achieve this, we needed to grade publications. We decided to measure quality based on criteria defined in Table 2. The criteria are designed to measure the quality of the publication itself, as well as the relevance of the blockchain research in the publication. All of the final 33 publications were independently investigated by three reviewers.

Table 2. Parameters for Measuring Quality.

\begin{tabular}{lll}
\hline ID & Quality Assessment Query & Quality Indicator (0-2) \\
\hline Q1 Is the publication relevant to blockchain? & BARELY-PARTIALLY-SATISFACTORILY \\
\hline Q2 & $\begin{array}{l}\text { Does the publication include and define research } \\
\text { objectives adequately? }\end{array}$ & NO-PARTIALLY-YES \\
\hline Q3 & Are limitations and challenges well defined? & NO-PARTIALLY- YES \\
\hline Q4 & Is the proposed contribution well described? & NO-PARTIALLY-YES \\
\hline
\end{tabular}

Each criterion was posed in the form of a question for the reviewers to answer. The reviewers could answer the questions with three answers varying from a negative answer when the criterion was addressed very poorly or not at all, to an intermediary response when the criterion was partially addressed, or a positive answer when the reviewer felt the publication had successfully satisfied the criterion. The answers were assigned values 0 ('BARELY' or 'NO'), 1 ('PARTIALLY'), and 2 ('SATISFACTORILY' or 'YES') and summarized across the reviewers to get final quality indicator scores (from 0 to 6 ) for each of the publications, where a higher score represents a better fulfillment of the criteria.

\section{Analysis}

\subsection{Bibliometric Overview}

We firstly present a bibliometric overview of selected publications in Figure 2. For the systematic review to be complete we also included grey literature, such as working papers and white papers. 
Just five publications are highly cited (e.g., more than 10 citations per publication). These publications were published in 2016 and 2017 at two conferences and in three journals. The reason for low citation numbers is that most publications were published in the last two years (2017 and 2018). Because they are relatively new there was not enough time to gather citations and we can assume that those publications will get additional citations in the future. All the publications with 0 citation were published in 2017 and 2018 and all the publications with 16 or more citations were published in 2016 .

Of the final 33 publications, 14 are conference proceeding papers, 11 are journal papers, 3 are patents and 5 papers are grey literature. Regarding the publication type, most publications in 2017 were published in peer-reviewed conference proceedings. This paradigm has shifted in 2018 as most publications were published in peer-reviewed journals. Figure 2 also shows that the overall amount of research has declined in 2018 when compared to the year before. However, please note that the data for 2018 is only for the first half of the year and that the number of published publications has already almost reached the number from 2017 and by far exceeded the number from 2016. Given the current number of publications for 2018 we have no doubt that the number of publications will again exceed the preceding year. This shows that the research field of blockchain in healthcare is very much relevant and growing.

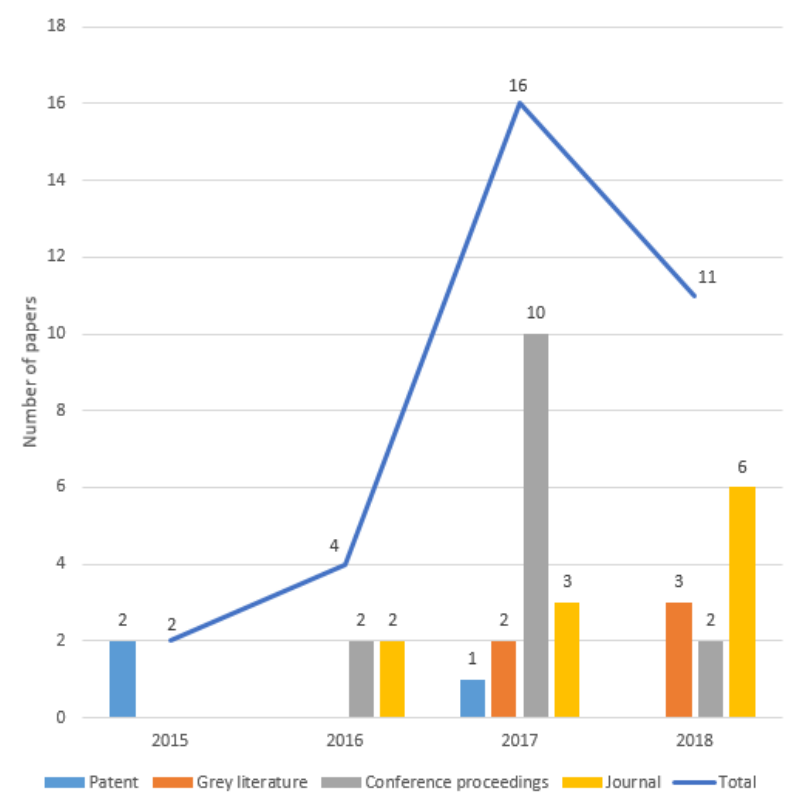

Figure 2. Number of publications published per year based on publication type.

\subsection{Analysis of Data Gathering}

Table 3 contains basic information on the analyzed publications, which are the publication's ID, publication reference (Reference), year of publication (Year), total number of citations (Citation Counter), in what kind of publication it was published (Publication Type) and the publisher (Publisher). The vast majority of research was published in peer-reviewed journals or in peer-reviewed conference proceedings. The final selection also includes a few patents (which are also reviewed before publication) and grey literature which was included for completeness. Table 3 shows that most of these publications have citations, and the ones that have none are newer (i.e., 2017 and 2018). 
Table 3. Bibliometric overview of selected articles.

\begin{tabular}{|c|c|c|c|c|c|}
\hline Publication ID & Reference & Year & Citation Counter & Publication Type & Publisher \\
\hline 1 & [43] & 2018 & 2 & Journal & IEEE Access \\
\hline 2 & [44] & 2017 & 0 & Conference & $\begin{array}{l}\text { IEEE International Symposium on } \\
\text { Autonomous Decentralized System (ISADS) }\end{array}$ \\
\hline 3 & [45] & 2018 & 0 & Conference & $\begin{array}{l}\text { IEEE EMBS International Conference on } \\
\text { Biomedical Health Informatics (BHI) }\end{array}$ \\
\hline 4 & [46] & 2017 & 0 & Conference & $\begin{array}{l}\text { International Conference on Software, } \\
\text { Telecommunications and Computer } \\
\text { Networks (SoftCOM) }\end{array}$ \\
\hline 5 & [47] & 2017 & 9 & Conference & $\begin{array}{l}\text { IFIP/IEEE Symposium on Integrated } \\
\text { Network and Service Management (IM) }\end{array}$ \\
\hline 6 & [48] & 2017 & 2 & Conference & $\begin{array}{l}\text { IEEE Annual International Symposium on } \\
\text { Personal, Indoor, and Mobile Radio } \\
\text { Communications (PIMRC) }\end{array}$ \\
\hline 7 & [49] & 2017 & 0 & Conference & $\begin{array}{l}\text { IEEE International Conference on e-Health } \\
\text { Networking, Applications and Services } \\
\text { (Healthcom) }\end{array}$ \\
\hline 8 & [50] & 2016 & 16 & Journal & IEEE Access \\
\hline 9 & [51] & 2016 & 95 & Conference & $\begin{array}{l}\text { International Conference on Open and Big } \\
\text { Data (OBD) }\end{array}$ \\
\hline 10 & [52] & 2017 & 3 & Conference & $\begin{array}{l}\text { IEEE International Conference on e-Health } \\
\text { Networking, Applications and Services } \\
\text { (Healthcom) }\end{array}$ \\
\hline 11 & [28] & 2017 & 5 & Journal & J. Biomed. Inform. \\
\hline 12 & [53] & 2018 & 1 & Journal & Health Informatics Journal \\
\hline 13 & [54] & 2017 & 8 & Conference & AMIA Symposium \\
\hline 14 & [55] & 2016 & 68 & Journal & Journal of Medical Systems \\
\hline 15 & [56] & 2017 & 2 & Journal & JMIR Mhealth And Uhealth \\
\hline 16 & [57] & 2018 & 0 & Journal & $\begin{array}{l}\text { International Journal of Distributed Sensor } \\
\text { Networks }\end{array}$ \\
\hline 17 & [58] & 2017 & 15 & Journal & Information \\
\hline 18 & [59] & 2018 & 2 & Journal & Sustainable Cities and Society \\
\hline 19 & [60] & 2018 & 0 & Journal & Journal of Medical Systems \\
\hline 20 & [61] & 2017 & 3 & Conference & $\begin{array}{l}\text { Security, Privacy, and Anonymity in } \\
\text { Computation, Communication, and Storage }\end{array}$ \\
\hline 21 & [62] & 2016 & 14 & Conference & NIST Workshop Blockchain Healthcare \\
\hline 22 & [63] & 2018 & 6 & Grey literature & arXiv \\
\hline 23 & [64] & 2017 & 8 & Grey literature & arXiv \\
\hline 24 & [65] & 2017 & 3 & Grey literature & Patientory.com \\
\hline 25 & [66] & 2015 & 2 & Patent & US patent \\
\hline 26 & [67] & 2017 & 2 & Conference & $\begin{array}{l}\text { IEEE International Conference on Big Data } \\
\text { (Big Data) }\end{array}$ \\
\hline 27 & [68] & 2015 & 3 & Patent & US patent \\
\hline 28 & [69] & 2017 & 0 & Conference & Norsk Informasjonssikkerhetskonferanse \\
\hline 29 & [70] & 2018 & 0 & Grey literature & University of Southampton \\
\hline 30 & [71] & 2018 & 0 & Grey literature & arXiv \\
\hline 31 & [72] & 2018 & 0 & Conference & $\begin{array}{l}\text { Workshop em Blockchain: Teoria, } \\
\text { Tecnologias e Aplicacoes }\end{array}$ \\
\hline 32 & [73] & 2017 & 0 & Patent & US patent \\
\hline 33 & [74] & 2018 & 0 & Journal & Int. J. Environ. Res. Public Health \\
\hline
\end{tabular}

Altogether we have analyzed 33 publications using the methodology mentioned in Section 3.4.

Table 4 shows the publications' contributions. Out of the 33 publications, a new structural design (i.e., introducing a framework, an architecture or a model employing a blockchain) occurred $27(82 \%)$ times. Algorithms, protocols or schemes were proposed in $4(12 \%)$ publications. The remaining $2(6 \%)$ publications present two other contributions-a new consensus algorithm and metrics for benchmarking distributed applications based on a blockchain platform.

The next analysis that we conducted in our systematic review is the purpose or field of blockchain use in healthcare. As can be seen in Table 5, most publications apply blockchain in healthcare for data sharing, health records and access control. Often, authors state multiple applications of blockchain technology in healthcare (e.g., for data sharing and access control) which could be regarded as expected since the blockchain technology itself implies specific applications-e.g., it is the nature of a distributed 
technology like blockchain to be used for data sharing, and it is therefore understandable that this field of research would be often mentioned.

Table 4. Contributions in the publication.

\begin{tabular}{cc}
\hline & Number of Publications \\
\hline Structural design & 27 \\
Algorithm/protocol & 4 \\
New consensus algorithm & 1 \\
Metric & 1 \\
\hline
\end{tabular}

Table 5. Field of blockchain application in healthcare.

\begin{tabular}{cc}
\hline Field & Number of Publications \\
\hline data sharing & 20 \\
health records & 18 \\
access control & 15 \\
audit trail & 6 \\
supply chain & 2 \\
other & 8 \\
\hline
\end{tabular}

After collecting data on the realization of the ideas described in publications we have discovered that the majority of publications rarely presented a working solution, although this trend has started to turn as we can see in Figure 3. Since 2017 there were five publications that presented a working solution, while there were none in the years before that. Since our search was done in June of 2018, there will probably be more publications with implemented solutions in 2018 and in the future.

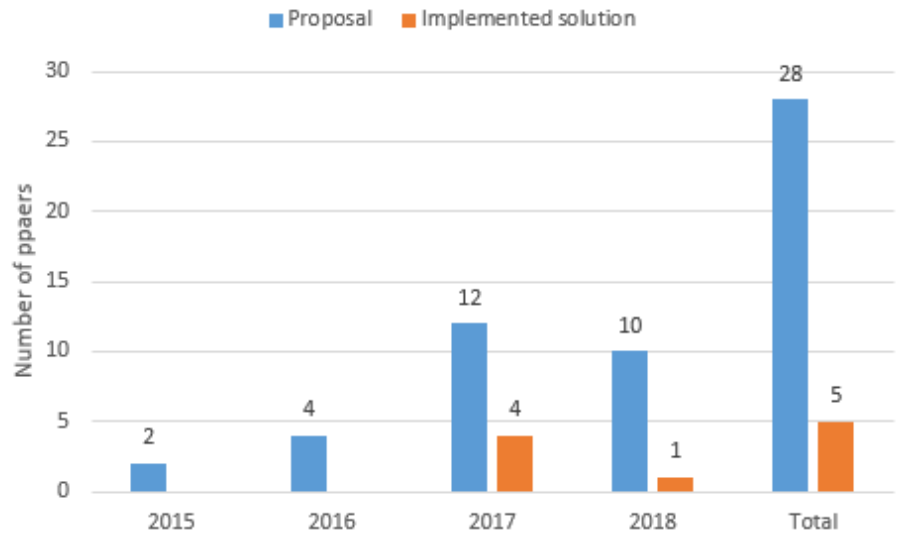

Figure 3. Number of papers published per year presenting a proposal or solution that is already implemented.

We planned to collect technical details pertaining to blockchain from the selected publications; however, we have discovered that these are very poorly described. The blockchain elements are typically poorly defined or not defined at all. The absence of details can be attributed to the fact that most publications are highly theoretical and do not include a prototype or a real-life implementation. Nevertheless, we gathered information on blockchain platforms and consensus algorithms where possible. Additionally, we have tried to identify the types of blockchains used and to ascertain if the use of a smart contract was proposed or not. Please note that not all blockchain platforms support smart contracts-for example, the Ethereum platform supports smart contracts, whereas Bitcoin does not.

As can be observed from Table 6, the platforms which are most commonly used are Ethereum and Hyperledger Fabric while the most common consensus algorithm defined in the publications is the proof-of-work. None of the publications used a public blockchain platform, but rather a public 
permissioned (consortium) or a private type of blockchain. Almost half of the analyzed publications also proposed the use of smart contracts for their solutions.

Table 6. Number of papers for each blockchain elements defined in the publications.

\begin{tabular}{|c|c|c|}
\hline & & No. of Publications \\
\hline \multirow{4}{*}{ Blockchain platform } & Ethereum & 10 \\
\hline & Hyperledger Fabric & 5 \\
\hline & Gcoin & 1 \\
\hline & not defined & 15 \\
\hline \multirow{7}{*}{ Consensus algorithm } & proof-of-work & 7 \\
\hline & proof-of-information & 1 \\
\hline & proof-of-interoperability & 1 \\
\hline & proof-of-stake & 1 \\
\hline & Practical Byzantine Fault Tolerance & 1 \\
\hline & QuorumChain & 1 \\
\hline & not defined & 21 \\
\hline \multirow{3}{*}{ Type } & private & 5 \\
\hline & consortium (public permissioned) & 6 \\
\hline & not defined & 22 \\
\hline \multirow{2}{*}{ Smart contracts } & yes & 15 \\
\hline & not defined & 18 \\
\hline
\end{tabular}

\subsection{Literature Quality Analysis}

The final and most important part of our systematic review was the scoring by the reviewers to measure the quality of the analyzed publications and the relevancy of blockchain usage, i.e., how blockchain-centered the described research actually is. The used methodology is presented in Section 3.5.

Table 7 shows the results of this scoring. Each query from Q1 to Q4 was assessed by 3 reviewers which resulted in a minimum of 0 points and a maximum of 6 points per query. The minimum for the column 'Q2-Q4' (a sum of Q2, Q3, and Q4) was 0 and the maximum was 18 points. Additionally, we created an overall score, where we combined the scores from Q1 to Q4, so that Q1 was $40 \%$ of the total value, and Q2 to Q4 were $20 \%$ of the total points each. To improve readability and comprehension, in the column 'Overall score Q1-Q4' a percentage like score is presented (number of points out of a maximum of 100). Please note that the explanation of the scoring for the queries $\mathrm{Q} 1$ to $\mathrm{Q} 4$ is given in Table 2.

In Figure 4 the average overall score per publication type per year is presented as well as a trendline, showing the average overall score for all the papers published in each year. In the years 2015 and 2016, fewer types of publications were published with a relatively high score. In the year 2017, 16 publications were published, but as can be observed their overall quality slightly fell when compared to the year before. The most recent 11 publications published in 2018 improved in quality.

In Figure 5 the score for each publication (distinct publication ID) is presented. Here a more detailed analysis and overall scores are presented. The average overall score is:

- 54.6 for 14 conference papers;

- 54.0 for 5 grey literature papers;

- 69.0 for 3 patents and

- 64.5 for 11 journal papers.

The results show a higher quality of patents and journal papers in comparison with the conference and grey literature papers. However, it has to be noted that the differences are not high. It is also interesting that based on Figure 4 the quality of conference papers is slightly on the decline while the journal paper's overall quality is slightly better each year. 
Table 7. Summary of scores for measuring quality of the publications.

\begin{tabular}{|c|c|c|c|c|c|c|}
\hline \multirow{2}{*}{ ID } & \multicolumn{4}{|c|}{ Summary of Points per Query } & \multirow{2}{*}{ Q2-Q4 } & \multirow{2}{*}{$\begin{array}{c}\text { Overall Score } \\
\text { Q1-Q4 }\end{array}$} \\
\hline & Q1 & Q2 & Q3 & Q4 & & \\
\hline 1 & 2 & 3 & 0 & 5 & 8 & 40 \\
\hline 2 & 0 & 3 & 0 & 5 & 8 & 27 \\
\hline 3 & 3 & 4 & 2 & 4 & 10 & 53 \\
\hline 4 & 3 & 5 & 2 & 4 & 11 & 57 \\
\hline 5 & 5 & 5 & 0 & 6 & 11 & 70 \\
\hline 6 & 4 & 3 & 1 & 5 & 9 & 57 \\
\hline 7 & 2 & 3 & 1 & 2 & 6 & 33 \\
\hline 8 & 3 & 4 & 2 & 6 & 12 & 60 \\
\hline 9 & 6 & 5 & 4 & 6 & 15 & 90 \\
\hline 10 & 4 & 5 & 3 & 3 & 11 & 63 \\
\hline 11 & 2 & 5 & 2 & 5 & 12 & 53 \\
\hline 12 & 6 & 5 & 5 & 5 & 15 & 90 \\
\hline 13 & 3 & 3 & 0 & 4 & 7 & 43 \\
\hline 14 & 4 & 4 & 2 & 4 & 10 & 60 \\
\hline 15 & 5 & 5 & 3 & 6 & 14 & 80 \\
\hline 16 & 4 & 2 & 2 & 3 & 7 & 50 \\
\hline 17 & 4 & 3 & 1 & 4 & 8 & 53 \\
\hline 18 & 6 & 6 & 3 & 6 & 15 & 90 \\
\hline 19 & 4 & 3 & 6 & 4 & 13 & 70 \\
\hline 20 & 4 & 3 & 0 & 3 & 6 & 47 \\
\hline 21 & 5 & 2 & 0 & 3 & 5 & 50 \\
\hline 22 & 4 & 3 & 3 & 4 & 10 & 60 \\
\hline 23 & 5 & 3 & 2 & 3 & 8 & 60 \\
\hline 24 & 3 & 2 & 2 & 3 & 7 & 43 \\
\hline 25 & 5 & 4 & 3 & 5 & 12 & 73 \\
\hline 26 & 5 & 2 & 0 & 5 & 7 & 57 \\
\hline 27 & 5 & 4 & 1 & 5 & 10 & 67 \\
\hline 28 & 5 & 4 & 0 & 6 & 10 & 67 \\
\hline 29 & 5 & 4 & 0 & 6 & 10 & 67 \\
\hline 30 & 3 & 3 & 0 & 3 & 6 & 40 \\
\hline 31 & 3 & 3 & 3 & 3 & 9 & 50 \\
\hline 32 & 5 & 4 & 0 & 6 & 10 & 67 \\
\hline 33 & 4 & 5 & 1 & 5 & 11 & 63 \\
\hline
\end{tabular}

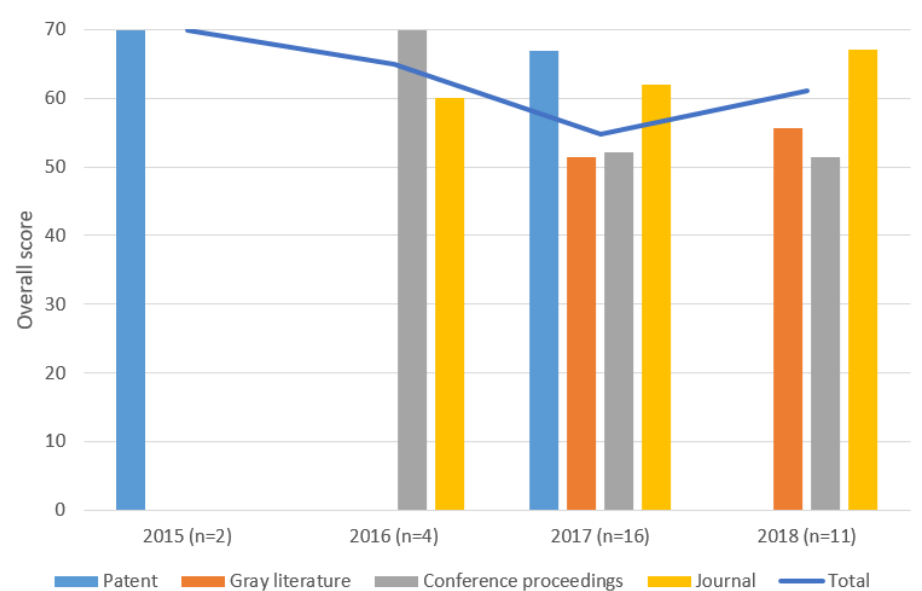

Figure 4. Average overall score per publication and year. 


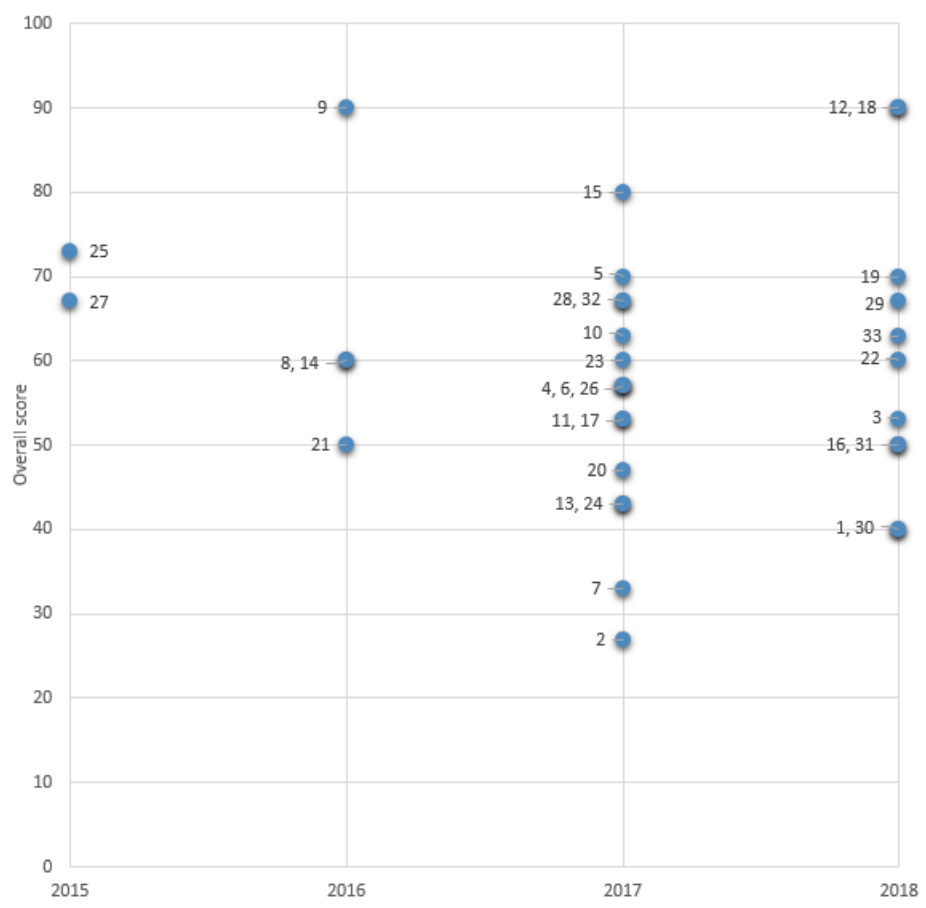

Figure 5. Overall score for each publication (ID) per year.

\section{Discussion}

In this section, we present a discussion on the retrieved data from the analyzed publications. We proposed three research questions which are discussed here.

RQ1: To what extent is a blockchain established in healthcare and how did this change over time?

In this systematic review, we searched publications published between 2008 and 2019 on blockchain technology used in healthcare. The publications that were identified as suitable for analysis were published after 2015, indicating that the technology is new and just entering the healthcare field. In the review process, we identified 33 publications, out of which only 5 presented an implemented and deployed solution as can been seen in Figure 3. In most publications, proposals are made that could be implemented, but mostly were not. The implemented solutions were published in 2017 and 2018, which indicates that interest in employing blockchain in healthcare is rising.

Another indicator of blockchain being used in healthcare through time shows that more publications are published each year on this topic, as presented in Figure 2. Our search was done in June 2018 and therefore only the first half of 2018 is included. In this period, 11 publications were published on the topic of blockchain in healthcare. In the year 2015, only 2 patents were published, while in the year 2016 there were 4 publications. Most publications (16) were published in 2017. The results of this study showed that the use of blockchain in healthcare is increasing and does not show any sign of waning.

RQ2: What are the current research trends for blockchain use in healthcare?

The areas of healthcare addressed in the analyzed publications demonstrate current research trends in this field. The results presented in Table 5, indicate that most of the publications use blockchain technology for the purpose of data sharing, health records and access control. Additionally, blockchain is rarely used for supply chain management, audit trail management and other scenarios, like drug prescription management and auditing. We could argue that blockchain as a decentralized system is therefore reasonably used (as to Würst and Gervais decision model [40]) in the analyzed publications in the field of healthcare. However, with regard to the European Coordination Committee 
of the Radiological and Electromedical and Healthcare IT Industry [39] there are still many possibilities for employing blockchain in healthcare, like medical billing or anti-counterfeiting drugs.

RQ3: What are the elements of blockchain technology used in healthcare publications?

An analysis of contributions was conducted and we also looked at blockchain elements that were used or proposed to be used in the publications. Most of the publications presented a structural design such as a framework, an architecture or a model for use in healthcare and employing a blockchain (Table 4). Far fewer publications presented a new algorithm or a protocol. Additionally, one publication presented a new consensus algorithm and one a metric for benchmarking healthcare distributed apps (dApp). When searching for specific blockchain elements that were used or suggested in the research we found that authors very rarely specified them (Table 6). This could either be because the research we have found is highly theoretical or because these are all components that can be switched to better suit one's needs and the authors did not wish to prescribe a sin-gular solution. Nevertheless, the publications that did specify the blockchain elements have shown Ethereum and Hyper-ledger Fabric to be the most popular blockchain platforms. The 11 publications that defined the blockchain type used a private or a consortium (public permissioned) blockchain. This is expected for healthcare as it is desirable to have control over access and writing to the blockchain and it is not desirable that writing (adding blocks to the blockchain) could be done by anyone. The most commonly used consensus algorithm turned out to be proof-of-work. This was very surprising to us. Healthcare is an environment where the speed of transactions could be very important and PoW is a very slow consensus algorithm. PoW is also generally not used with consortium or private chains where the actors are generally known (in this case, hospitals, patients, insurance companies, etc.) and trust is easier to establish. Consortium or private chains are usually more centralized (e.g., hospitals) than public networks where anybody can be a node and where PoW is the dominant consensus algorithm. Finally, the PoW is also a very computationally demanding algorithm. It would be impractical if hospitals would have to establish large computer centres just to mine the transactions. For all of these reasons we were surprised to see the PoW being used this often. We expected algorithms like proof-of-authority or proof-of-stake to be much more popular, because they fit around all the discussed PoW limitations. We suspect that PoW was so popular in the gathered research, because as we have seen in the previous section the use of blockchain in healthcare is only getting started and the pioneers in the field looked to existing solutions for inspiration. The most common and publicized application of blockchain are the cryptocurencies where PoW is prevalent. Additionally, 15 publications contained (explicitly) the intent of using smart contracts in their proposal. It is unexpected to note that less than half of all proposals did not use an element as useful as a smart contract. Smart contracts enable additional functionalities and new possibilities of blockchain application in healthcare-e.g., we can limit access, automate certain tasks, etc.

In summary, the elements of blockchain used in the gathered research is broad, as would be expected. Researchers build networks with some participant restrictions (consortium and private networks), to give the network better control over access and record creation. The two most commonly used platforms in the selected publications are probably the two most popular and well developed platforms-Ethereum and Hyperledger Fabric, both of which support smart contracts. Smart contracts add a lot of functionality to a blockchain, but researchers do not seem to be using them quite as often as one would think. Therefore, a further use of smart contracts and the introduction of less constraining consensus algorithms would be, in our opinion, the primary area for further research and better integration of blockchain technology into healthcare.

\section{Conclusions and Future Work}

This study investigated the current blockchain research trends in healthcare. The blockchain technology presents a decentralized network and is regarded as having great potential for use in healthcare, because of the sensitive nature of data being processed and managed. The aim of the study 
was to identify the current status of blockchain research and application in healthcare. To achieve this objective, we have defined research questions and using the predefined methodology we have narrowed down the analyzed literature to 33 publications. These were then further analyzed. We have searched 9 relevant online databases for publications published between 2008 and 2019.

For the in-depth analysis, the set of 33 publications was inspected by three reviewers. We have collected data as prompted by our research questions and assessed the publications using the predefined assessment criteria.

Our findings indicate that blockchain technology research and its employment in healthcare is increasing. Current trends of blockchain research in healthcare indicate that it is mostly used for data sharing, health records and access control, but rarely for other scenarios, such as supply chain management or drug prescription management. Therefore, much potential for blockchain is still unexploited. Most re-search presents a novel framework, architecture or model using blockchain technology in healthcare. Additionally, often technical details about the used blockchain elements are not given-e.g., blockchain platform, consensus algorithm, blockchain type or the use of smart contracts. Particularly, smart contracts could be more used as they enable the automation of processes within a blockchain platform. Most research could also provide a prototype implementation or at least discuss some implementation details of their proposals.

As for further research, blockchains are still fairly new technology in the field of healthcare and new ways for employing it can still be found and researched. To sum up-blockchain should continue to be used in scenarios where it is reasonable and needed.

Author Contributions: All authors equally contributed to the conception of the idea, the layout of the research plan, the construction of the search queries and participated in the literature search. M.H. led research activities, designed the conceptualization, contributed to the investigation, defined and reviewed the methodology, performed the validation and co-wrote the original and revised versions of the paper; M.K. contributed to the investigation, performed the validation and co-wrote the original and revised versions of the paper; A.K. performed the validation and co-wrote the original and revised versions of the paper; L.N.Z. contributed to the investigation, defined the visualization, performed the validation and co-wrote the original and revised versions of the paper; All authors equally contributed to the rest of the paper; All authors read the manuscript and approved its content.

Funding: The authors acknowledge financial support from the Slovenian Research Agency (Research Core Funding No. P2-0057).

Acknowledgments: The authors acknowledge financial support from the Slovenian Research Agency (Research Core Funding No. P2-0057).

Conflicts of Interest: The authors declare no conflict of interest. 


\section{Appendix A}

Table A1. Systematic Review Protocol.

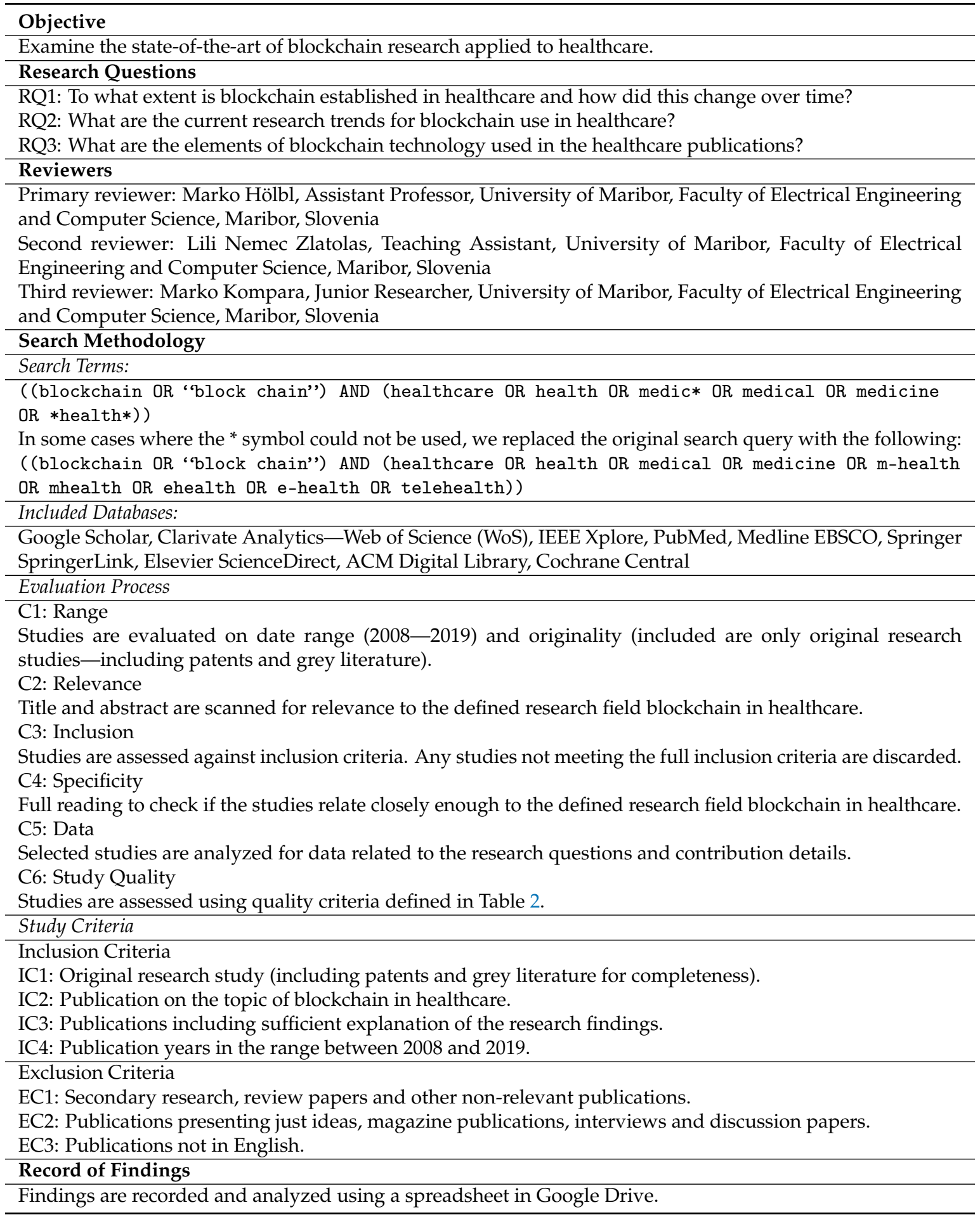

\section{References}

1. McKinsey \& Company. Blockchain Technology in the Insurance Sector. In Proceedings of the Quarterly Meeting of the Federal Advisory Committee on Insurance (FACI), New York, NY, USA, 5 January 2017.

2. Crypto-Currency Market Capitalization. 2018. Available online: https://coinmarketcap.com/ (accessed on 4 September 2018). 
3. Nakamoto, S. Bitcoin: A Peer-To-Peer Electronic Cash System; Portal Unicamp: Campinas, Brazil, 2008.

4. Swan, M. Blockchain: Blueprint for a New Economy; O’Reilly Media: Newton, MA, USA, 2015.

5. Singh, S.; Singh, N. Blockchain: Future of financial and cyber security. In Proceedings of the 2016 2nd International Conference on Contemporary Computing and Informatics (IC3I), Noida, India, 14-17 December 2016; pp. 463-467. [CrossRef]

6. Tschorsch, F.; Scheuermann, B. Bitcoin and Beyond: A Technical Survey on Decentralized Digital Currencies. IEEE Commun. Surv. Tutor. 2016, 18, 2084-2123. [CrossRef]

7. Ethereum. Ethereum Project; Ethereum Switzerland GmbH (EthSuisse): Zug, Switzerland, 2015.

8. Christidis, K.; Devetsikiotis, M. Blockchains and Smart Contracts for the Internet of Things. IEEE Access 2016, 4, 2292-2303. [CrossRef]

9. Zheng, Z.; Xie, S.; Dai, H.; Chen, X.; Wang, H. An Overview of Blockchain Technology: Architecture, Consensus, and Future Trends. In Proceedings of the 2017 IEEE International Congress on Big Data (BigData Congress), Boston, MA, USA, 11-14 December 2017; pp. 557-564. [CrossRef]

10. Park, J.; Park, J. Blockchain Security in Cloud Computing: Use Cases, Challenges, and Solutions. Symmetry 2017, 9, 164. [CrossRef]

11. Yin, S.; Bao, J.; Zhang, Y.; Huang, X. M2M security technology of CPS based on blockchains. Symmetry 2017, 9, 193. [CrossRef]

12. Karafiloski, E.; Mishev, A. Blockchain solutions for big data challenges: A literature review. In Proceedings of the IEEE EUROCON 2017-17th International Conference on Smart Technologies, Ohrid, Macedonia, 6-8 July 2017; pp. 763-768. [CrossRef]

13. Ahram, T.; Sargolzaei, A.; Sargolzaei, S.; Daniels, J.; Amaba, B. Blockchain technology innovations. In Proceedings of the 2017 IEEE Technology Engineering Management Conference (TEMSCON), Santa Clara, CA, USA, 8 June 2017; pp. 137-141. [CrossRef]

14. Conoscenti, M.; Vetro, A.; Martin, J.C.D. Blockchain for the Internet of Things: A systematic literature review. In Proceedings of the 2016 IEEE/ACS 13th International Conference of Computer Systems and Applications (AICCSA), Agadir, Morocco, 29 November-2 December 2016; pp. 1-6. [CrossRef]

15. Yli-Huumo, J.; Ko, D.; Choi, S.; Park, S.; Smolander, K. Where Is Current Research on Blockchain Technology?-A Systematic Review. PLoS ONE 2016, 11, e0163477. [CrossRef] [PubMed]

16. Tama, B.A.; Kweka, B.J.; Park, Y.; Rhee, K.H. A critical review of blockchain and its current applications. In Proceedings of the 2017 International Conference on Electrical Engineering and Computer Science (ICECOS), Palembang, Indonesia, 22-23 August 2017; pp. 109-113. [CrossRef]

17. Mettler, M. Blockchain technology in healthcare: The revolution starts here. In Proceedings of the 2016 IEEE 18th International Conference on e-Health Networking, Applications and Services (Healthcom), Munich, Germany, 14-17 September 2016; pp. 1-3. [CrossRef]

18. Zhang, P.; Schmidt, D.C.; White, J.; Lenz, G. Blockchain Technology Use Cases in Healthcare. In Advances in Computers; Elsevier: Amsterdam, The Netherlands, 2018. [CrossRef]

19. Kuo, T.T.; Kim, H.E.; Ohno-Machado, L. Blockchain distributed ledger technologies for biomedical and health care applications. J. Am. Med. Inform. Assoc. 2017, 24, 1211-1220. [CrossRef] [PubMed]

20. Angraal, S.; Krumholz, H.M.; Schulz, W.L. Blockchain Technology: Applications in Health Care. Circ. Cardiovasc. Qual. Outcomes 2017, 10, e003800. [CrossRef] [PubMed]

21. Mackey, T.K.; Nayyar, G. A review of existing and emerging digital technologies to combat the global trade in fake medicines. Expert Opin. Drug Saf. 2017, 16, 587-602. [CrossRef] [PubMed]

22. Giungato, P.; Rana, R.; Tarabella, A.; Tricase, C. Current Trends in Sustainability of Bitcoins and Related Blockchain Technology. Sustainability 2017, 9, 2214. [CrossRef]

23. Engelhardt, M. Hitching Healthcare to the Chain: An Introduction to Blockchain Technology in the Healthcare Sector. Technol. Innov. Manag. Rev. 2017, 7, 22-34. [CrossRef]

24. Alhadhrami, Z.; Alghfeli, S.; Alghfeli, M.; Abedlla, J.A.; Shuaib, K. Introducing blockchains for healthcare. In Proceedings of the 2017 International Conference on Electrical and Computing Technologies and Applications (ICECTA), Ras Al Khaimah, UAE, 21-23 November 2017; pp. 1-4. [CrossRef]

25. Sater, S. Blockchain Transforming Healthcare Data Flows. SSRN Electr. J. 2018. [CrossRef]

26. Aste, T.; Tasca, P.; Di Matteo, T. Blockchain Technologies: The Foreseeable Impact on Society and Industry. Computer 2017, 50, 18-28. [CrossRef] 
27. Raval, S. Decentralized Applications: Harnessing Bitcoin's Blockchain Technology, 1st ed.; O’Reilly Media, Inc.: Sebastopol, CA, USA, 2016.

28. Roehrs, A.; da Costa, C.A.; da Rosa Righi, R.; Alex, R.; Costa, C.A.; Righi, R.R. OmniPHR: A distributed architecture model to integrate personal health records. J. Biomed. Inform. 2017, 71, 70-81. [CrossRef] [PubMed]

29. Sleiman, M.D.; Lauf, A.P.; Yampolskiy, R. Bitcoin Message: Data Insertion on a Proof-of-Work Cryptocurrency System. In Proceedings of the 2015 International Conference on Cyberworlds (CW), Visby, Sweden, 7-9 October 2015; pp. 332-336. [CrossRef]

30. Aumasson, J. Serious Cryptography: A Practical Introduction to Modern Encryption; No Starch Press: San Francisco, CA, USA, 2017.

31. Ferguson, N.; Schneier, B. Practical Cryptography, 1st ed.; John Wiley \& Sons, Inc.: New York, NY, USA, 2003.

32. National Institute of Standards and Technology. Secure Hash Standard (SHS); Federal Information Processing Standards Publication: Gaithersburg, MD, USA, 2012.

33. Greenspan, G. Blockchains Vs Centralized Databases; MultiChain: London, UK, 2016.

34. Lewis, A. A Gentle Introduction to Blockchain Technology. Bits on Blocks. 2018. Available online: https:// bitsonblocks.net/2015/09/09/gentle-introduction-blockchain-technology/ (accessed on 4 September 2018).

35. Litecoin. Litecoin-Open Source P2P Digital Currency. 2013. Available online: https://litecoin.org/ (accessed on 4 September 2018).

36. Androulaki, E.; Manevich, Y.; Muralidharan, S.; Murthy, C.; Nguyen, B.; Sethi, M.; Singh, G.; Smith, K.; Sorniotti, A.; Stathakopoulou, C.; et al. Hyperledger fabric: A distributed operating system for permissioned blockchains. In Proceedings of the Thirteenth EuroSys Conference, Porto, Portugal, 23-26 April 2018. [CrossRef]

37. Ripple. Ripple-One Frictionless Experience To Send Money Globally. 2018. Available online: https:/ / ripple. com/ (accessed on 4 September 2018).

38. Borge, M.; Kokoris-Kogias, E.; Jovanovic, P.; Gasser, L.; Gailly, N.; Ford, B. Proof-of-Personhood: Redemocratizing Permissionless Cryptocurrencies. In Proceedings of the 2017 IEEE European Symposium on Security and Privacy Workshops (EuroS\&PW), Paris, France, 26-28 April 2017; pp. 23-26. [CrossRef]

39. European Coordination Committee of the Radiological. Blockchain in Healthcare; Technical report; European Coordination Committee of the Radiological: Brussels, Belgium, 2017.

40. Wüst, K.; Gervais, A. Do you need a Blockchain? IACR Cryptol. ePrint Arch. 2017, 2017, 375.

41. CASP UK. CASP Checklists-CASP-Critical Appraisal Skills Programme. Qual. Res. Checkl. 2017, $31,449$.

42. Google Scholar Citations. Available online: https://scholar.google.com/citations (accessed on 4 September 2018).

43. Guo, R.; Shi, H.; Zhao, Q.; Zheng, D. Secure Attribute-Based Signature Scheme With Multiple Authorities for Blockchain in Electronic Health Records Systems. IEEE Access 2018, 6, 11676-11686. [CrossRef]

44. Zhao, H.; Zhang, Y.; Peng, Y.; Xu, R. Lightweight Backup and Efficient Recovery Scheme for Health Blockchain Keys. In Proceedings of the 2017 IEEE 13th International Symposium on Autonomous Decentralized System (ISADS), Bangkok, Thailand, 22-24 March 2017; pp. 229-234. [CrossRef]

45. Juneja, A.; Marefat, M. Leveraging blockchain for retraining deep learning architecture in patient-specific arrhythmia classification. In Proceedings of the 2018 IEEE EMBS International Conference on Biomedical Health Informatics (BHI), Las Vegas, NV, USA, 4-7 March 2018; pp. 393-397. [CrossRef]

46. Angeletti, F.; Chatzigiannakis, I.; Vitaletti, A. The role of blockchain and IoT in recruiting participants for digital clinical trials. In Proceedings of the 2017 25th International Conference on Software, Telecommunications and Computer Networks (SoftCOM), Split, Croatia, 21-23 September 2017; pp. 1-5. [CrossRef]

47. Bocek, T.; Rodrigues, B.B.; Strasser, T.; Stiller, B. Blockchains everywhere-A use-case of blockchains in the pharma supply-chain. In Proceedings of the 2017 IFIP/IEEE Symposium on Integrated Network and Service Management (IM), Lisbon, Portugal, 8-12 May 2017; pp. 772-777. [CrossRef]

48. Liang, X.; Zhao, J.; Shetty, S.; Liu, J.; Li, D. Integrating blockchain for data sharing and collaboration in mobile healthcare applications. In Proceedings of the 2017 IEEE 28th Annual International Symposium on Personal, Indoor, and Mobile Radio Communications (PIMRC), Montreal, QC, Canada, 8-13 October 2017; pp. 1-5. [CrossRef]

49. Liu, W.; Zhu, S.S.; Mundie, T.; Krieger, U. Advanced block-chain architecture for e-health systems. In Proceedings of the 2017 IEEE 19th International Conference on e-Health Networking, Applications and Services (Healthcom), Dalian, China, 12-15 October 2017; pp. 1-6. [CrossRef] 
50. Zhang, J.; Xue, N.; Huang, X. A Secure System For Pervasive Social Network-Based Healthcare. IEEE Access 2016, 4, 9239-9250. [CrossRef]

51. Azaria, A.; Ekblaw, A.; Vieira, T.; Lippman, A. MedRec: Using Blockchain for Medical Data Access and Permission Management. In Proceedings of the 2016 2nd International Conference on Open and Big Data (OBD), Vienna, Austria, 22-24 August 2016; pp. 25-30. [CrossRef]

52. Zhang, P.; Walker, M.A.; White, J.; Schmidt, D.C.; Lenz, G. Metrics for assessing blockchain-based healthcare decentralized apps. In Proceedings of the 2017 IEEE 19th International Conference on e-Health Networking, Applications and Services (Healthcom), Dalian, China, 12-15 October 2017; pp. 1-4. [CrossRef]

53. Patel, V. A framework for secure and decentralized sharing of medical imaging data via blockchain consensus. Health Inform. J. 2018, 1460458218769699. [CrossRef] [PubMed]

54. Dubovitskaya, A.; Xu, Z.; Ryu, S.; Schumacher, M.; Wang, F. Secure and Trustable Electronic Medical Records Sharing using Blockchain. AMIA Annu. Symp. Proc. 2017, 2017, 650-659. [PubMed]

55. Yue, X.; Wang, H.; Jin, D.; Li, M.; Jiang, W. Healthcare Data Gateways: Found Healthcare Intelligence on Blockchain with Novel Privacy Risk Control. J. Med. Syst. 2016, 40, 218. [CrossRef] [PubMed]

56. Ichikawa, D.; Kashiyama, M.; Ueno, T. Tamper-Resistant Mobile Health Using Blockchain Technology. JMIR mHealth uHealth 2017, 5, e111. [CrossRef] [PubMed]

57. Lee, S.H.; Yang, C.S. Fingernail analysis management system using microscopy sensor and blockchain technology. Int. J. Distrib. Sens. Netw. 2018, 14, 1550147718767044. [CrossRef]

58. Xia, Q.; Sifah, E.B.; Smahi, A.; Amofa, S.; Zhang, X. BBDS: Blockchain-based data sharing for electronic medical records in cloud environments. Information 2017, 8, 44. [CrossRef]

59. Dagher, G.G.; Mohler, J.; Milojkovic, M.; Marella, P.B. Ancile: Privacy-preserving framework for access control and interoperability of electronic health records using blockchain technology. Sustain. Cities Soc. 2018, 39, 283-297. [CrossRef]

60. Griggs, K.N.; Ossipova, O.; Kohlios, C.P.; Baccarini, A.N.; Howson, E.A.; Hayajneh, T. Healthcare Blockchain System Using Smart Contracts for Secure Automated Remote Patient Monitoring. J. Med. Syst. 2018, 42, 130. [CrossRef] [PubMed]

61. Al Omar, A.; Rahman, M.S.; Basu, A.; Kiyomoto, S.; Wang, G.; Atiquzzaman, M.; Yan, Z.; Choo, K.K.R. MediBchain: A Blockchain Based Privacy Preserving Platform for Healthcare Data; Springer: Cham, Switzerland, 2017; pp. 534-543.

62. Peterson, K.; Deeduvanu, R.; Kanjamala, P.; Boles, K. A blockchain-based approach to health information exchange networks. Proc. NIST Workshop Blockchain Healthc. 2016, 1, 1-10.

63. Kuo, T.T.; Hsu, C.N.; Ohno-Machado, L. ModelChain: Decentralized Privacy-Preserving Healthcare Predictive Modeling Framework on Private Blockchain Networks. NIST Workshop Blockchain Healthc. 2016, 26-27. [CrossRef]

64. Zhang, P.; White, J.; Schmidt, D.C.; Lenz, G. Applying Software Patterns to Address Interoperability in Blockchain-based Healthcare Apps. arXiv 2017, arXiv:1706.03700.

65. Mcfarlane, C.; Beer, M.; Brown, J.; Prendergast, N. Patientory: A Healthcare Peer-to-Peer EMR Storage Network v1.1; Entrust Inc.: Addison, TX, USA, 2017.

66. Witchey, N.J. Healthcare Transaction Validation Via Blockchain Proof-of-Work, Systems and Methods. U.S. Patent US20150332283A1, 19 November 2015.

67. Lemieux, V.L. A typology of blockchain recordkeeping solutions and some reflections on their implications for the future of archival preservation. In Proceedings of the 2017 IEEE International Conference on Big Data (Big Data 2017), Boston, MA, USA, 11-14 December 2017; IEEE: Piscataway, NJ, USA, 2018; pp. 2271-2278. [CrossRef]

68. Tanner, T.; Dunlevy, T.S. System and Method for Decentralized Autonomous Healthcare Economy Platform. U.S. Patent US20170039330A1, 9 February 2017.

69. Yang, H.; Yang, B. A Blockchain-based Approach to the Secure Sharing of Healthcare Data. In Proceedings of the Norwegian Information Security Conference 2017, Oslo, Norway, 27-29 November 2017.

70. Massi, M.; Miladi, A.; Margheri, A.; Sassone, V.; Rosenzweig, J. Using PROV and Blockchain to Achieve Health Data Provenance; Technical Report; University of Southampton Institutional Repository: Southampton, UK, 2018.

71. Dias, J.P.; Reis, L.; Ferreira, H.S.; Martins, Â. Blockchain for Access Control in e-Health Scenarios. arXiv 2018, arXiv:1805.12267. 
72. Conceição, A.F.; da Silva, F.S.C.; Rocha, V.; Locoro, A.; Barguil, J.M. Eletronic Health Records using Blockchain Technology. arXiv 2018, arXiv:1804.10078.

73. Bulleit, D.A.; Aluri, B.C.; Danner, F.T., III; Miller, C.C., III; Braunstein, M. Blockchain-Based Mechanisms for Secure Health Information Resource Exchange. U.S. Patent US20180060496A1, 1 March 2018.

74. Tseng, J.H.; Liao, Y.C.; Chong, B.; Liao, S.W. Governance on the Drug Supply Chain via Gcoin Blockchain. Int. J. Environ. Res. Public Health 2018, 15, 1055. [CrossRef] [PubMed]

(C) 2018 by the authors. Licensee MDPI, Basel, Switzerland. This article is an open access article distributed under the terms and conditions of the Creative Commons Attribution (CC BY) license (http://creativecommons.org/licenses/by/4.0/). 